

\title{
Material Selection Shape Factors for Compliant Arrays in Bending
}

\author{
Todd G. Nelson ${ }^{\mathrm{a}, *}$, Jared T. Bruton ${ }^{\mathrm{a}}$, Nathan E. Rieske ${ }^{\mathrm{a}}$, M. Patrick Walton ${ }^{\mathrm{a}}$, David T. \\ Fullwood $^{\mathrm{a}}$, Larry L. Howell ${ }^{\mathrm{a}}$ \\ ${ }^{a}$ Brigham Young University, Department of Mechanical Engineering, 435 CTB, Provo, UT 84602, United \\ States
}

\begin{abstract}
Similar to the general class of metamaterials, compliant arrays (CAs) are engineered from an array of subelements that combine to produce a response that is typically not available from a flat panel made of a single material. As such, analysis and design of CA systems requires the integration of both the material and geometrical properties of the array. This paper proposes a convenient and efficient method of combining these essential elements using analytically derived shape factors for bending modes. The approach is validated experimentally, and used to demonstrate large regions of previously inaccessible property combinations in material selection charts that become attainable using carefully design CAs.
\end{abstract}

Keywords: Shape factor, Bending, Material selection, Compliant, Stiffness, Metamaterials

\section{Introduction}

Compliant Arrays (CAs) are panels with geometry consisting of a fundamental unit that is patterned in parallel and series to achieve target material properties, particularly out-ofplane bending stiffness. An example of a CA in acrylic is shown in Figure 1. Similar to metamaterials, CAs create responses typically not achievable from a flat panel of a single material. They can be potentially manufactured in micro, meso, or macro scales. Many candidates for the fundamental unit of these arrays come from the field of Lamina Emergent Mechanisms (LEMs). LEMs are mechanical devices fabricated from planar materials (laminae) with motion that emerges out of the fabrication plane [1]. CAs lend themselves towards applications such as deployable or collapsible structures as they enable a larger elastic deformation in out-of-plane bending than is possible with a solid sheet for a given material. Recent developments with the use of patterns similar to those investigated in this paper include an array for solar tracking [2], flexible lithium-ion batteries [3], an optical shutter [4], the creation of super-conformable materials for applications such as flexible circuits [5], and creating materials with extreme Poisson's ratios $[6,7]$. CAs can also be modified to create thick-material approximations of curved-crease origami patterns used in origami-inspired

\footnotetext{
*Corresponding author

Email address: toddgn@byu.edu (Todd G. Nelson)
} 


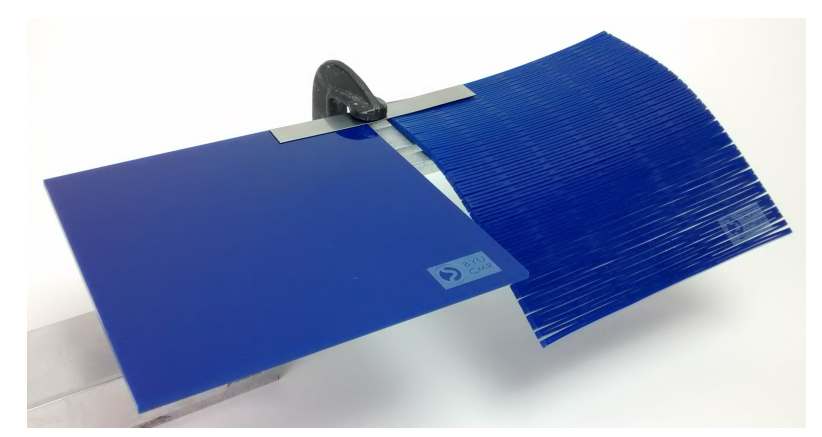

Figure 1: An acrylic compliant array (CA), shown on the right, bending under its own weight with a reference panel of same material, thickness, and area shown on the left.

mechanisms and structures [8]. While the body of work investigating stiff and light panels is extensive and ongoing through a variety of avenues [9-19], there is significantly less research describing methods to create panels with a lower, yet selectable, stiffness to enable large amounts of elastic deflection. Recent informative work by Ohshima et al. investigates the analysis and design of slit-based materials [20], which can also be classified as a type of CA. Isobe et al. also characterize motions of a kirigami pattern which could be considered a CA $[21]$.

Shape factors were developed to show the effect of a variable cross section for beams [22]. Also known as a shape transformer, a shape factor is defined as the ratio of a geometric quantity for a specific cross section over the same geometric quantity of a reference cross section $[23,24]$. Shape factors can be used to compare properties of a component or structure as a combination of geometry and material simultaneously. They provide a way to represent the properties of a shaped component as a "new'material through modification of the material properties. Comparison to other materials and geometries is then facilitated in the material selection process. Elastic bending shape factors have been previously developed for a variety of cross sections $[25,26]$, yet shape factors have not been developed to describe the impact of panels with patterns that can be described by planar geometry, such as those found in CAs. This research characterizes elastic bending shape factors and strength efficiency shape factors associated with two types of CAs. These factors function as design guidelines in selecting materials for applications requiring large magnitudes of elastic bending.

\section{Background}

\subsection{Compliance}

CAs rely on the fundamental principles of compliant mechanisms to function. Compliant mechanisms "gain at least some of their mobility from the deflection of flexible members rather than from movable joints only" [27]. Using compliant mechanisms to achieve the lamina-emergent function has several advantages over using traditional mechanisms, including simplified manufacturing, reduced wear, and reduced part count. In many cases, a lamina-emergent mechanism can have a part count of one, a quality retained by a CA. Because a CA consists of a single part, it can also be viewed as a modified material rather than 
strictly a mechanism. For a CA to function, the array must be able to deflect without failing by undergoing some combination of torsion and/or bending. As such, a fundamental unit consisting of different members that bend or twist can be developed to be used in arrays. Multiple individual units that can bend or deflect elastically to a certain displacement can be arrayed in series to achieve the desired elastic range of motion.

\subsection{CA Applications: Deployable Structures and Developable Surfaces}

Because CAs can be designed to achieve a large elastic range of motion, they are good candidates to be incorporated into deployable structures. Deployable structures have been used in space technology [28], portable shelters [29], microelectromechanical systems (MEMS) [30], outdoor equipment, and furniture. They come in a variety of sizes, shapes, and materials. These structures are commonly composed of developable surfaces because of planar manufacturing or storage requirements. While developable surfaces can be rigorously described by mathematics [31-33] and design guidelines for specific deployable applications exist [34], limited investigation of guidelines for material selection for deployable mechanisms has been conducted. Characterizing the elastic bending and strength efficiency shape factors of CAs provides material selection guidelines that can be incorporated into bending applications and deployable structure design.

CAs are particularly suited for transitioning curved-fold origami into thicker materials to create mechanisms and structures. This is because curved-fold origami results in a combination of curved surfaces joined by creases which Solomon [35] classifies as a general developable surface. While much of the field of curved folding is unexplored, several recent papers have presented design guidelines utilizing principles from optics for the creation of developable curved-fold designs [36-38]. The transition between paper-thin curved-fold origami to thicker engineering materials presents a unique set of challenges, including the high bending stresses introduced by the large possible curvatures of the surfaces. The majority of origami designs that have been transitioned to engineering materials fall into the category of rigidly foldable [39] as seen in the self-folding design of Felton et al. [40], where the panels do not flex or bend, thus facilitating physical construction. CAs can provide a path to creating curved-fold designs in thick materials [8].

\subsection{Shape Factors and Material Selection}

The concept of dimensionless shape factors and how they can be incorporated into material selection was first investigated by Ashby [22]. These shape factors enable designers to see the effect of shape on effective material properties. For example, an extruded aluminum I-beam can be considered a "new" material that is stiffer and stronger than a square aluminum beam. Shape factors describe how the material properties, such as modulus and yield strength, can be modified to achieve the effective properties of the "new" material. This paper will examine two shape factors in particular. The elastic bending shape factor for bending about a particular axis, $\phi_{B}^{e}$, is used to describe relative stiffness gain for a shape as compared to a reference shape of the same area and material. This shape factor can be expressed as [25] 


$$
\phi_{B}^{e}=\frac{S}{S_{o}}
$$

where $S$ is the stiffness of the shape and $S_{o}$ is the stiffness of the reference shape. For example, steel I-beams can have elastic bending shape factors ranging from approximately 1 to 65 [41]. This can be interpreted as the I-beam being a "new" material which has an effective modulus that is 1 to 65 times as stiff as the modulus of the material for a square beam of an equivalent cross-sectional area.

Another shape factor, the strength efficiency shape factor $\phi_{B}^{f}$, is a measure of how much stronger a shape is in bending compared to a reference shape of the same area and material. The strength efficiency shape factor is described [25] by

$$
\phi_{B}^{f}=\frac{M_{\max }}{M_{o, \max }}
$$

where $M_{\max }$ is the bending moment at failure of the shape and $M_{o, \max }$ is the bending moment at failure of the reference shape. Similarly, an I-beam made of aluminum of cross-sectional area, $A$, with a strength efficiency shape factor of 5 can be considered a "new" material with an effective yield strength 5 times as large as the yield strength of a square aluminum beam of the same cross-sectional area.

Shape factors have been characterized for many common cross-sectional shapes of beams [25]. Elastic bending shape factors for solid cross sections of circles, squares, and other regular polygons are typically close to one. Elongated, hollow, and I-shaped cross sections can have elastic bending shape factors of 50 or more. It is rare to find real components with shape factors above 65 due to manufacturing constraints and the onset of local buckling as the predominant failure mode [41]. The possible range of shape factors can also vary significantly from material to material. Several methods exist to incorporate shape factors into material selection processes. These include the method developed by Birmingham [42] to link material property charts with shape-efficiency charts, developing material indices (measures of material performance in a specific engineering situation) with shape factors included, and modifying material property charts by plotting a "new" material that is possible with shape factors. [25] The application of material indices to facilitate material selection has recently been introduced into the field origami-inspired structures, as seen in the research by Tolman et al. [43]

\section{Methods}

\subsection{Compliant Array Geometry}

The geometry of CAs can vary greatly as CAs are formed by linking together multiple units of a lamina emergent mechanism or multiple types of lamina emergent mechanisms. In this study two particular CAs have been chosen to illustrate general methods for CA shape factor analysis. These two CAs also demonstrate how a reduction in out-of-plane bending stiffness can be achieved in particular directions determined by the geometry of the array. The bending stiffness of an array about a particular axis, $K_{\text {array,axis }}$, will be defined such 

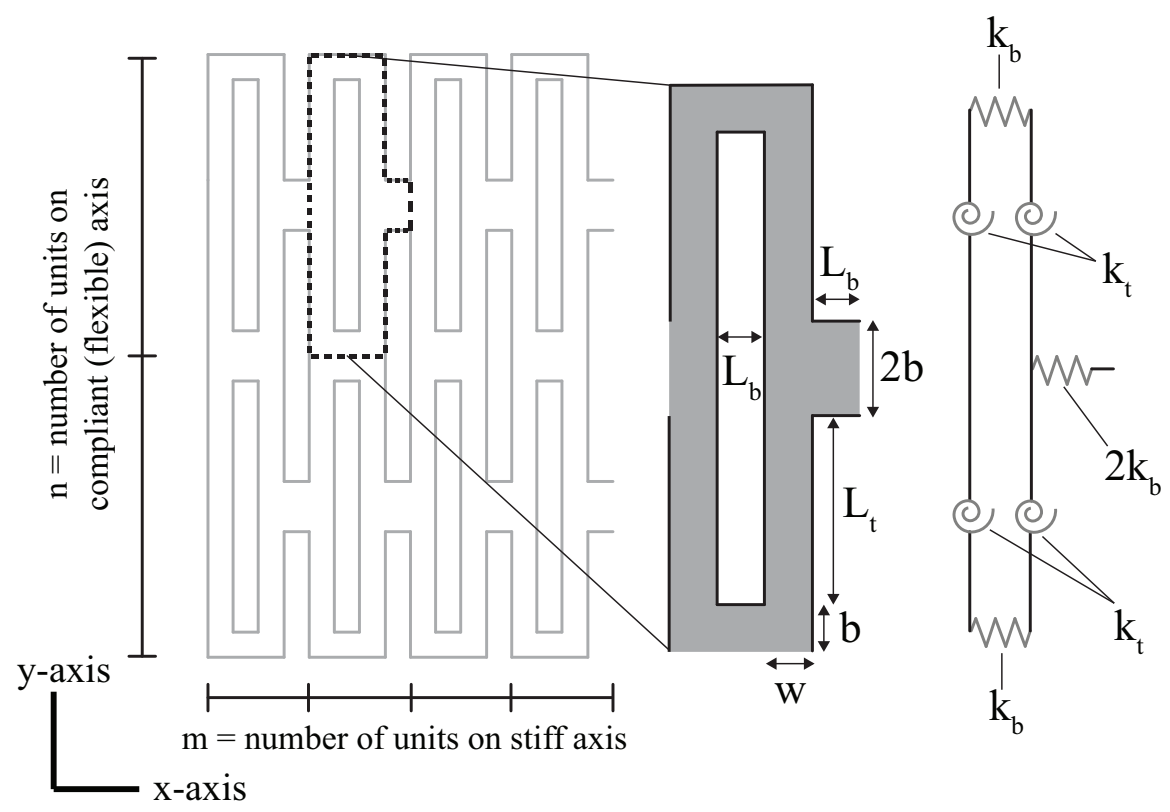

Figure 2: The Unidirectional CA geometry and parameters with a detailed fundamental unit and its corresponding spring model for bending across the y-axis.

that the angular rotation about the axis, $\Theta$, is related to the moment applied across the axis, $M$, by the equation

$$
M=K_{\text {array,axis }} \Theta
$$

\subsubsection{Unidirectional $C A$}

The Unidirectional CA is formed with the Outside Lamina Emergent Torsion joint (Outside LET) [44] as the fundamental unit, as illustrated in Figure 2. This array maintains a stiff bending axis, the x-axis, which can be nearly as stiff as a solid panel. It also enables a compliant bending axis offset by 90 degrees from the stiff bending axis, the y-axis. Its name, Unidirectional CA, is derived from this property of having one bending direction that sees a marked decrease in stiffness. When a moment is applied about the flexible axis, the y-axis, the four legs function as torsion bars. When a moment is applied about the stiff axis, the $\mathrm{x}$-axis, the fundamental unit's four legs function as bending beams, which can retain much of the original solid panel's stiffness.

The stiffness of the Outside LET unit is characterized using a model similar to the torsion spring model developed by Jacobsen et al. [45] The bending stiffness about the y-axis, $k_{y}$, can be expressed using the geometry definitions of Figure 2 as

$$
k_{y}=\frac{k_{b} k_{t}}{k_{b}+k_{t}}
$$

or if $k_{b}$ is much greater than $k_{t}$ (at least two orders of magnitude) 


$$
k_{y}=k_{t} \quad \text { if } k_{b} \gg k_{t}
$$

The spring constant of the torsion legs, $k_{t}$, equivalent to a rectangular beam in torsion [46], is expressed as

$$
k_{t}=\frac{G w t^{3}\left(\frac{1}{3}-0.21 \frac{t}{w}\left(1-\frac{t^{4}}{12 w^{4}}\right)\right)}{L_{t}}
$$

where $t$ is the thickness of the sheet, $w \geq t$, and $G$ is the shear modulus. This symbolic expression for the torsional stiffness of a rectangular beam where $w \geq t$ is an approximation of the infinite series solution derived from elasticity theory $[46,47]$. Comparisons of the approximate equation solution to the series solution show error of less a half a percent [45]. The spring constant of the bending regions, $k_{b}$, is given by

$$
k_{b}=\frac{E I}{L_{b}} \quad \text { with } \quad I=\frac{b t^{3}}{12}
$$

where $E$ is Young's modulus. This stiffness is used to relate the moment applied to the joint about the y-axis, $M$, and the resulting angular deflection of the joint about the y-axis, $\theta$, as

$$
M=k_{y} \theta
$$

These definitions for the fundamental unit can be extended to the Unidirectional CA by considering each fundamental unit as a torsion spring connected to the neighboring units in parallel and series. By letting $m$ be the number of units along the x-axis and $n$ be the number of units along the y-axis, the total stiffness of the Unidirectional CA across the y-axis, $K_{u n i, y}$, can be expressed as

$$
K_{u n i, y}=\left(\frac{n}{m}\right) k_{y}
$$

As explained in Equation 3, the total angular displacement of the array, $\Theta$, is related to the moment applied to the array about the compliant bending axis, $M$, by

$$
M=K_{\text {uni,y }} \Theta
$$

In a similar manner, the rotational stiffness of the fundamental unit about the $\mathrm{x}$-axis, $k_{x}$, can be expressed as

$$
k_{x}=\frac{E w\left(w+\frac{1}{2} L_{b}\right) t^{3}}{6\left(4 b w+2 w L_{t}+L_{b} L_{t}\right)}
$$

where $E$ is Young's modulus. This expression was derived by accounting for each leg of the fundamental unit as a spring in bending (as none of the legs act in torsion for loading across the $\mathrm{x}$-axis) and combining the legs together as springs in parallel and series to find an expression for the total rotational stiffness for bending around the $\mathrm{x}$-axis. The form for the bending stiffness of each leg resembles Equation 7, with variation in the expression for 


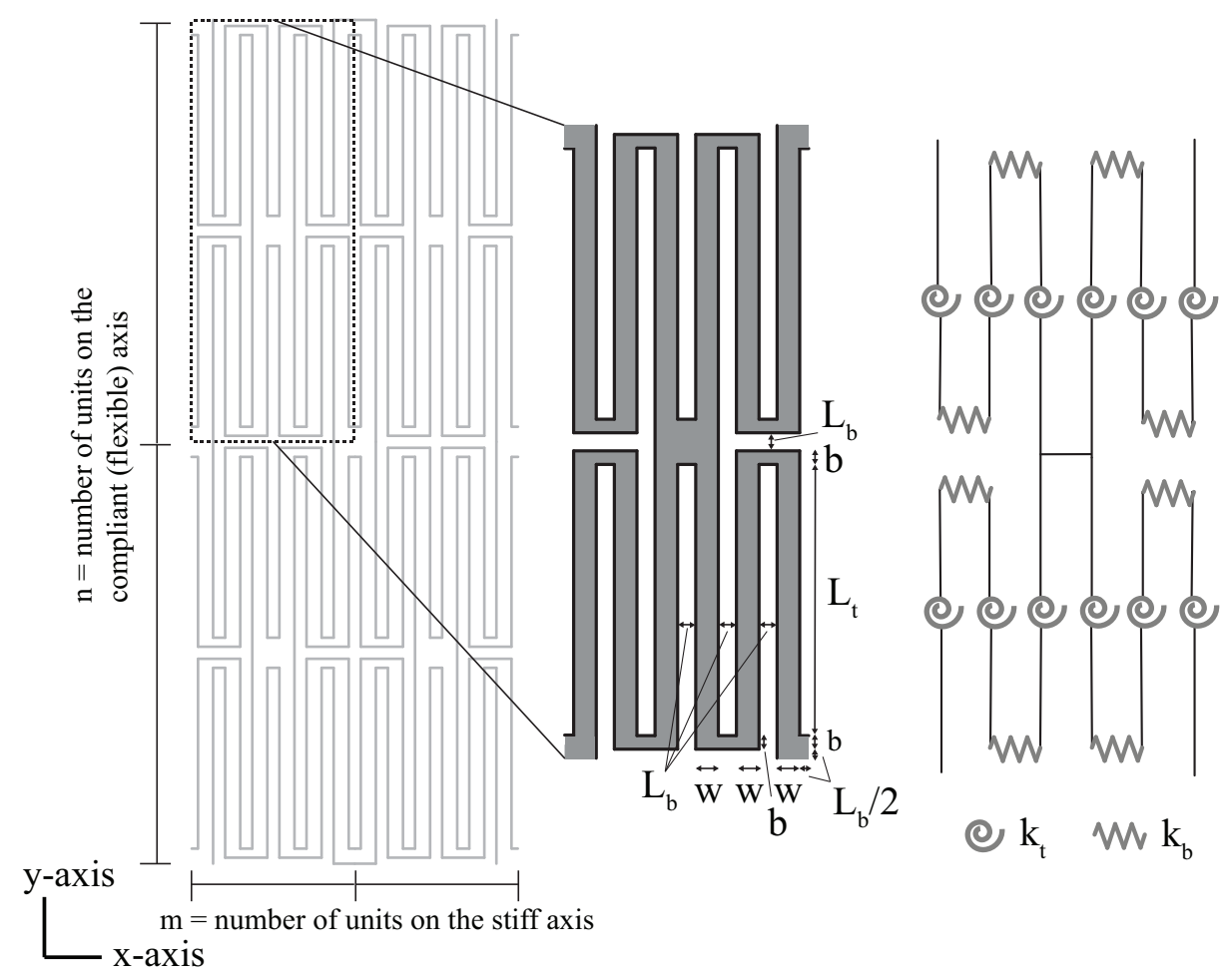

Figure 3: Bidirectional CA geometry and parameters with a detailed fundamental unit and its corresponding spring model for bending across the y-axis.

$I$ and in the denominator of the stiffness expression for $k_{b}$ corresponding to the geometry of each leg.

The total stiffness of the Unidirectional CA across the x-axis can be expressed as

$$
K_{u n i, x}=\left(\frac{m}{n}\right) k_{x}
$$

where once again $m$ is the number of units along the y-axis and $n$ is the number of units along the $\mathrm{x}$-axis.

\subsubsection{Bidirectional $C A$}

The Bidirectional CA uses a variation of the Outside LET joint as its fundamental unit to provide a reduction in stiffness in two orthogonal directions as shown in Figure 3. Across the compliant bending axis, the y-axis, the legs of the fundamental units function as torsion bars while across the stiffer bending axis, the x-axis, they are placed in bending.

The bending stiffness of the fundamental unit about the y-axis, $k_{y}$, for the Bidirectional CA can be expressed as

$$
k_{y}=\frac{k_{b} k_{t}}{3 k_{b}+2 k_{t}}
$$

or if $k_{b}$ is at least two orders of magnitude greater than $k_{t}$ 


$$
k_{y}=\frac{k_{t}}{3} \quad \text { if } \quad k_{b} \gg k_{t}
$$

where $k_{t}$ and $k_{b}$ are described by Equation 6 and Equation 7 . The middle portion of the fundamental unit is considered to be rigid for bending about the y-axis as it is stiffer than all other areas of the joint in this bending mode. Equation 8 describes the relationship of the angular deflection of the unit, the bending stiffness of the unit, and the moment applied to the unit.

The total stiffness of the Bidirectional CA across the y-axis, $K_{b i, y}$, can be expressed as

$$
K_{b i, y}=\left(\frac{n}{m}\right) k_{y}
$$

where $m$ represents the number of units along the x-axis and $n$ represents the number of units along the y-axis.

By modeling each leg of the joint as a spring, the expression for the bending stiffness of the fundamental unit of the Bidirectional CA across the x-axis, $k_{x}$, is

$$
k_{x}=\frac{E w\left(w+\frac{1}{2} L_{b}\right) t^{3}}{6\left(2 w b+3 L_{t}\left(L_{b}+2 w\right)+2 w\left(L_{b}+2 b\right)\right)}
$$

where $E$ is Young's modulus. Similar to the way Equation 11 was derived, this expression was found by considering each leg of the fundamental unit to be in bending, calculating the bending stiffness of each leg as is shown in Equation 7, and then combining the stiffnesses of all the legs together using rules for springs in parallel and series to find an equivalent total stiffness for the fundamental unit. It should be noted that the middle portion of the Bidirectional CA is not considered to be rigid for computing the stiffness across the $\mathrm{x}$-axis as it was for computing the stiffness across the y-axis. This is because the middle portion is no longer much stiffer compared to the other areas of the joint. The total stiffness of the Bidirectional CA across the x-axis, $K_{b i, x}$, can be expressed as

$$
K_{b i, x}=\left(\frac{m}{n}\right) k_{x}
$$

Examples of the Unidirectional and Bidirectional arrays were created through laser cutting $0.292 \mathrm{~cm}(0.115 \mathrm{in})$ thick acrylic and $0.114 \mathrm{~cm}(0.045 \mathrm{in})$ thick steel. These are shown in Figure 4.

\subsection{Analytical Characterization of Bending Shape Factors and Strength Efficiency Factors}

\subsubsection{Bending Shape Factors}

The shape factor and strength efficiency shape factor for the Unidirectional and Bidirectional CAs can be analytically determined for both the compliant and stiff bending axes. Using the elastic bending shape factor definition from Equation 1, the elastic bending shape factor for the Unidirectional array across the y-axis with a moment loading can be expressed as 


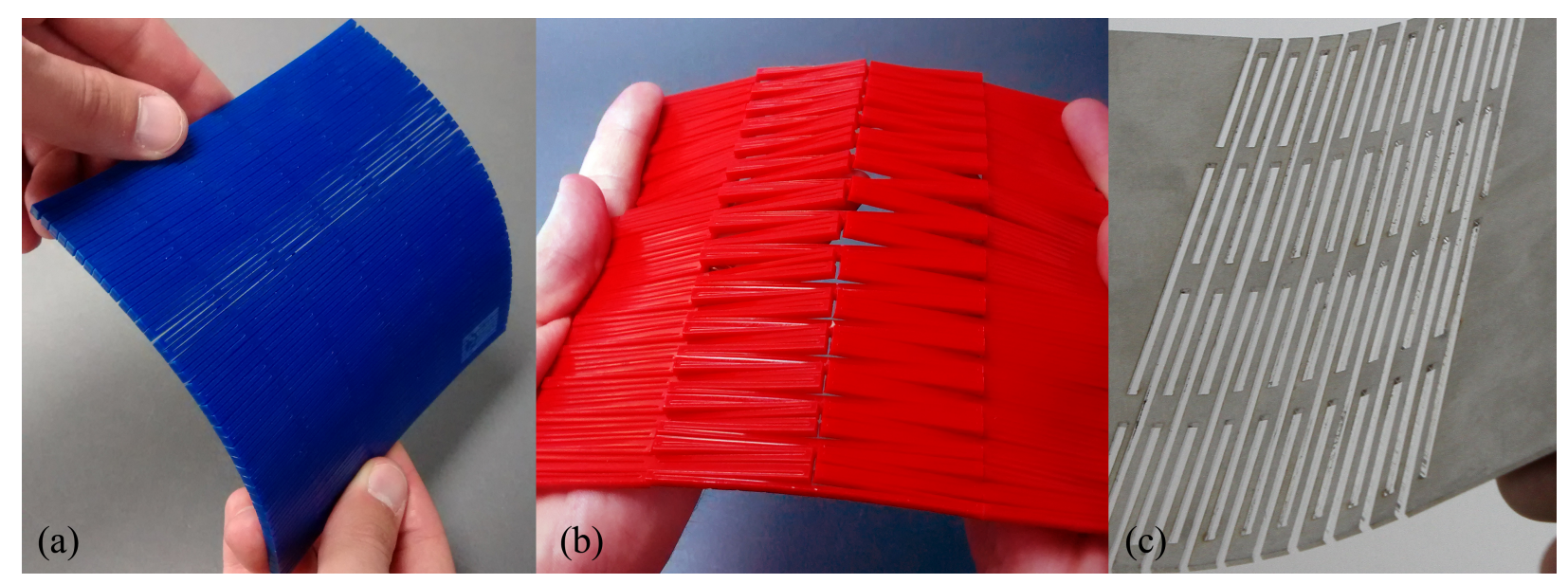

Figure 4: Examples of (a) Unidirectional CA in acrylic bending about its y (compliant) axis, (b) Bidirectional $\mathrm{CA}$ in acrylic bending about its $\mathrm{x}$ (stiff) axis, and (c) Unidirection CA in steel bending about its y (compliant) axis.

$$
\phi_{B}^{e}=\frac{S_{y}}{S_{o, y}}=\frac{K_{u n i, y}}{\frac{E I_{o, y}}{L_{o, y}}}
$$

where $I_{o, y}$ is the second moment of area for the cross section of the reference shape and is expressed as

$$
I_{o, y}=\frac{n\left(4 b+2 L_{t}\right) t^{3}}{12}
$$

and $L_{o, y}$ is the length of the reference shape perpendicular to the bending axis and is expressed as

$$
L_{o, y}=m\left(2 w+2 L_{b}\right)
$$

Typically, elastic bending shape factors are most useful when they are only a function of geometry. To minimize the number of properties affecting the shape factor, Equations 4, 6, 7,9 and

$$
G=\frac{E}{2(1+\nu)}
$$

are combined. Note that Equation 21 is only valid for isotropic materials where $G$ is the shear modulus, $E$ is Young's modulus, and $\nu$ is Poisson's ratio. Combining these equations results in

$$
\phi_{B}^{e}=\frac{\frac{6 b w\left(w+L_{b}\right)}{\left(2 b+L_{t}\right)}\left(\frac{1}{3}-0.21\left(\frac{t}{w}\right)\left(1-\frac{t^{4}}{12 w^{4}}\right)\right)}{(1+\nu) b L_{t}+6 L_{b} w\left(\frac{1}{3}-0.21\left(\frac{t}{w}\right)\left(1-\frac{t^{4}}{12 w^{4}}\right)\right)}
$$

If $k_{b} \gg k_{t}$, then the equation simplifies to 


$$
\phi_{B}^{e}=\frac{6 w\left(w+L_{b}\right)}{(1+\nu) L_{t}\left(2 b+L_{t}\right)}\left(\frac{1}{3}-0.21 \frac{t}{w}\left(1-\frac{t^{4}}{12 w^{4}}\right)\right)
$$

By approximating $\nu$ by 0.3 , which is common for metals and many isotropic materials [48], this equation can be expressed as

$$
\phi_{B}^{e}=\frac{4.615 w\left(w+L_{b}\right)}{L_{t}\left(2 b+L_{t}\right)}\left(\frac{1}{3}-0.21 \frac{t}{w}\left(1-\frac{t^{4}}{12 w^{4}}\right)\right)
$$

The elastic bending shape factor about the x-axis for the Unidirectional array can be found in a similar manner as follows

$$
\phi_{B}^{e}=\frac{S_{x}}{S_{o, x}}=\frac{K_{u n i, x}}{\frac{E I_{o, x}}{L_{o, x}}}
$$

where

$$
I_{o, x}=\frac{m\left(2 w+2 L_{b}\right) t^{3}}{12}
$$

and

$$
L_{o, x}=n\left(4 b+2 L_{t}\right)
$$

Substitutions from Equations 11 and 12 allow the shape factor about the x-axis for the Unidirectional array in bending to be written as a function of geometry as

$$
\phi_{B}^{e}=\frac{w\left(2 w+L_{b}\right)\left(2 b+L_{t}\right)}{\left(w+L_{b}\right)\left(4 b w+2 w L_{t}+L_{b} L_{t}\right)}
$$

The procedure for finding the elastic bending shape factor for the Bidirectional array is similar to that of the Unidirectional array. Similarly, elastic bending shape factors can be written for both arrays when $t \geq w$ by swapping $t$ and $w$ in Eq. 6 during the derivation. The elastic bending shape factors for both arrays are summarized in Table 1 for $w \geq t$ and in Table 2 for $t \geq w$.

\subsubsection{Strength Efficiency Shape Factors}

The strength efficiency shape factor as defined in Equation 2 can be found for a moment load across the y-axis for the Unidirectional CA with the following procedure. Using standard mechanics equations, the maximum bending moment of the reference panel, $M_{o, \max }$, can be expressed as

$$
M_{o, \max }=\frac{\sigma_{y} I_{o, y}}{c} \quad \text { with } \quad I_{o, y}=\frac{n\left(4 b+2 L_{t}\right) t^{3}}{12}
$$

where $c$ is the distance furthest from the neutral axis of the cross section of the panel and $\sigma_{y}$ is the yield stress of the material. 
Table 1: Bending Shape Factors for CAs with $w \geq t$.

\section{Unidirectional CA}

\begin{tabular}{|c|c|c|c|}
\hline \multirow{4}{*}{ Bending about y-axis, } & \multirow{4}{*}{$\phi_{B}^{e}$} & \multirow[t]{2}{*}{ In general, } & $\frac{6 b w\left(w+L_{b}\right)}{\left(2 b+L_{t}\right)}\left(\frac{1}{3}-0.21\left(\frac{t}{w}\right)\left(1-\frac{t^{4}}{12 w^{4}}\right)\right)$ \\
\hline & & & $(1+\nu) b L_{t}+6 L_{b} w\left(\frac{1}{3}-0.21\left(\frac{t}{w}\right)\left(1-\frac{t^{4}}{12 w^{4}}\right)\right)$ \\
\hline & & if $k_{b} \gg k_{t}$ & $\frac{6 w\left(w+L_{b}\right)}{(1+\nu) L_{t}\left(2 b+L_{t}\right)}\left(\frac{1}{3}-0.21 \frac{t}{w}\left(1-\frac{t^{4}}{12 w^{4}}\right)\right)$ \\
\hline & & if $\nu=0.3$ and $k_{b} \gg k_{t}$ & $\frac{4.615 w\left(w+L_{b}\right)}{L_{t}\left(2 b+L_{t}\right)}\left(\frac{1}{3}-0.21 \frac{t}{w}\left(1-\frac{t^{4}}{12 w^{4}}\right)\right)$ \\
\hline \multirow[t]{2}{*}{ Bending about $\mathrm{x}$-axis, } & $\phi_{B}^{e}$ & \multicolumn{2}{|c|}{$\frac{w\left(2 w+L_{b}\right)\left(2 b+L_{t}\right)}{\left(w+L_{b}\right)\left(4 b w+2 w L_{t}+L_{b} L_{t}\right)}$} \\
\hline & & \multicolumn{2}{|c|}{ Bidirectional CA } \\
\hline \multirow{4}{*}{ Bending about y-axis, } & \multirow{4}{*}{$\phi_{B}^{e}$} & \multirow{2}{*}{ In general, } & $\frac{66 w\left(w+L_{b}\right)}{\left(2 b+L_{t}+L_{b}\right)}\left(\frac{1}{3}-0.21\left(\frac{t}{w}\right)\left(1-\frac{t^{4}}{12 w^{4}}\right)\right)$ \\
\hline & & & $(1+\nu) b L_{t}+4 L_{b} w\left(\frac{1}{3}-0.21\left(\frac{t}{w}\right)\left(1-\frac{t^{4}}{12 w^{4}}\right)\right)$ \\
\hline & & if $k_{b} \gg k_{t}$ & $\frac{6 w\left(w+L_{b}\right)}{(1+\nu) L_{t}\left(2 b+L_{t}+L_{b}\right)}\left(\frac{1}{3}-0.21 \frac{t}{w}\left(1-\frac{t^{4}}{12 w^{4}}\right)\right)$ \\
\hline & & if $\nu=0.3$ and $k_{b} \gg k_{t}$ & $\frac{4.615 w\left(w+L_{b}\right)}{L_{t}\left(2 b+L_{t}+L_{b}\right)}\left(\frac{1}{3}-0.21 \frac{t}{w}\left(1-\frac{t^{4}}{12 w^{4}}\right)\right)$ \\
\hline Bending about $\mathrm{x}$-axis, & $\phi_{B}^{e}$ & $\overline{3(w+}$ & 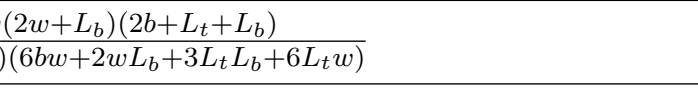 \\
\hline
\end{tabular}


Table 2: Bending Shape Factors for CAs with $t \geq w$.

\section{Unidirectional CA}

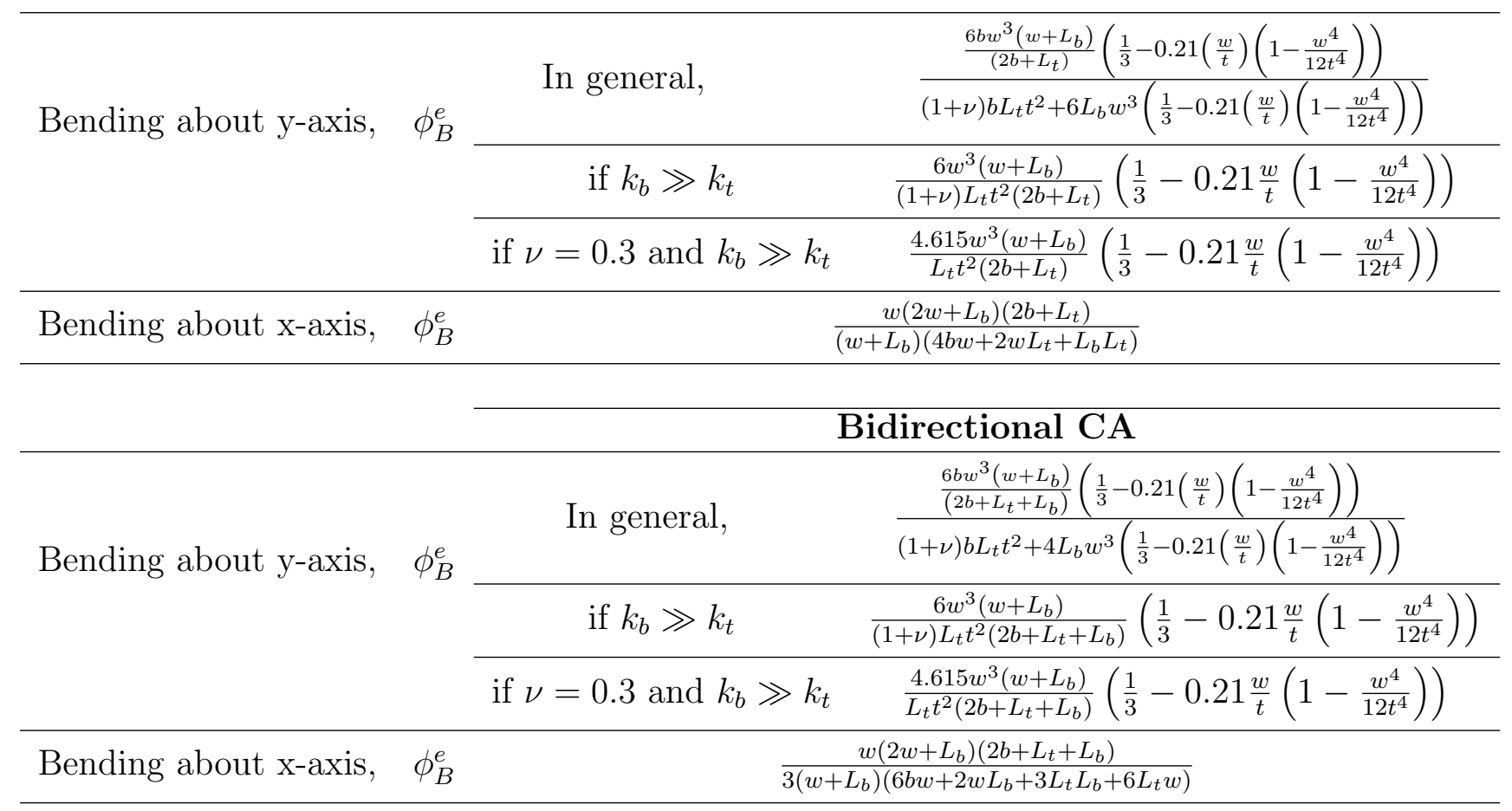


The maximum moment of the Unidirectional CA about the y-axis, $M_{\max , y}$, is equal to the maximum moment that one torsion bar of the fundamental unit can withstand before failure multiplied by $2 n$, as the maximum moment of the entire array is divided into load paths through $2 n$ torsion bars. This reasoning holds only when using ductile materials. With ductile materials the stress concentrations at the joints between the torsion and bending regions of the fundamental units can be disregarded as localized strengthening occurs from plastic strain. Thus for ductile materials the maximum moment the CA can withstand before failure can be expressed as [49]

$$
M_{\text {max }, y}=2 n \tau Q \quad \text { with } \quad Q=\frac{8\left(\frac{w t}{4}\right)^{2}}{3\left(\frac{w}{2}\right)+1.8\left(\frac{t}{2}\right)}
$$

where $\tau$ is the maximum shear stress of the material and $w \geq t$. If a brittle material is used to construct the CA, an appropriate stress concentration factor should be applied which will in effect reduce the value of $\tau$.

Substitution of Equations 29 and 30 into Equation 2 results in

$$
\phi_{B}^{f}=\frac{2 n \tau Q}{\frac{\sigma_{y} I_{o, y}}{c}}
$$

For this array $c=\frac{t}{2}$ and the approximation of $\tau=0.577 \sigma_{y}$ [49] can be used to simplify the equation to contain only geometric factors resulting in

$$
\phi_{B}^{f}=\frac{0.577 n Q t}{I_{o, y}}
$$

Further substitution for $I_{o, y}$ and $Q$ and simplifying gives the following expression of the strength efficiency shape factor for out-of-plane bending about the y-axis of the Unidirectional array where $w \geq t$.

$$
\phi_{B}^{f}=\frac{1.154 w^{2}}{(w+0.6 t)\left(2 b+L_{t}\right)}
$$

The strength efficiency shape factor for bending about the x-axis with a moment load for the Unidirectional array can similarly be found. The maximum moment of the reference panel, $M_{o, \max }$, can be expressed as

$$
M_{o, \max }=\frac{\sigma_{y} I_{o, x}}{c} \text { with } \quad I_{o, x}=\frac{m\left(2 w+2 L_{b}\right) t^{3}}{12}
$$

where $c$ is the distance furthest from the neutral axis of the cross section of the panel and $\sigma_{y}$ is the yield stress of the material. The maximum moment of the Unidirectional CA about the x-axis, $M_{\max , x}$, is equal to $2 m$ times the maximum moment which the bending bars of the fundamental unit can withstand before failure when using ductile materials. Beam mechanics equations can be used to express $M_{\max , x}$ as 
Table 3: Strength Efficiency Shape Factors for CAs with $w \geq t$.

\begin{tabular}{lccc} 
& & Unidirectional & Bidirectional \\
\hline Strength Efficiency about y-axis & $\left(\phi_{B}^{f}\right)$ & $\frac{1.154 w^{2}}{(w+0.6 t)\left(2 b+L_{t}\right)}$ & $\frac{0.3846 w^{2}}{(w+0.6 t)\left(2 b+L_{t}+L_{b}\right)}$ \\
Strength Efficiency about x-axis & $\left(\phi_{B}^{f}\right)$ & $\frac{w}{w+L_{b}}$ & $\frac{w}{3\left(w+L_{b}\right)}$ \\
\hline
\end{tabular}

Table 4: Strength Efficiency Shape Factors for CAs with $t \geq w$.

\begin{tabular}{lccc} 
& & Unidirectional & Bidirectional \\
\hline Strength Efficiency about y-axis & $\left(\phi_{B}^{f}\right)$ & $\frac{1.154 w^{2}}{(t+0.6 w)\left(2 b+L_{t}\right)}$ & $\frac{0.3846 w^{2}}{(t+0.6 w)\left(2 b+L_{t}+L_{b}\right)}$ \\
Strength Efficiency about x-axis & $\left(\phi_{B}^{f}\right)$ & $\frac{w}{w+L_{b}}$ & $\frac{w}{3\left(w+L_{b}\right)}$ \\
\hline
\end{tabular}

$$
M_{m a x, x}=\frac{2 m \sigma_{y} I_{u, x}}{c_{u}} \text { with } \quad I_{u, x}=\frac{w t^{3}}{12}
$$

where $\sigma_{y}$ is the yield stress of the material and $c_{u}$ is the distance furthest from the neutral axis of the cross section of the bending portion of the fundamental unit. Substituting Equations 34 and 35 into Equation 2 results in

$$
\phi_{B}^{f}=\frac{2 m I_{u, x} c}{I_{o, x} c_{u}}
$$

Making further substitutions for $I_{u, x}$ and $I_{o, x}$ and noting that $c=c_{u}=\frac{t}{2}$ for the array, the strength efficiency shape factor for the Unidirectional array with moment load about the $\mathrm{x}$-axis and $w \geq t$ can be expressed in geometrical parameters as

$$
\phi_{B}^{f}=\frac{w}{w+L_{b}}
$$

A similar procedure can be carried out to find the strength efficiency shape factors of the Bidirectional array about the $\mathrm{x}$ and $\mathrm{y}$ axes. In addition, expressions for strength efficiency factors for both arrays when $t \geq w$ can be found by swapping $t$ and $w$ in Eq. 30 during the derivation. The results are summarized in Table 3 for $w \geq t$ and in Table 4 for $t \geq w$.

\subsection{Experimental Verification}

Physical testing of the Unidirectional CA for bending about the y-axis was conducted to verify the method used to derive the shape factors described in this paper. Three unidirectional CA patterns were cut into $1.22 \mathrm{~mm}$ (0.048 in) thick stainless steel sheet using a water jet and described by the number of fundamental units used in the array $(m x n)$. Two prototypes, the $2 \times 2$ array and the $3 \times 2$ array, had all geometry of a fundamental unit the same (with small variation due to tolerances on the water jet). The third prototype was the 


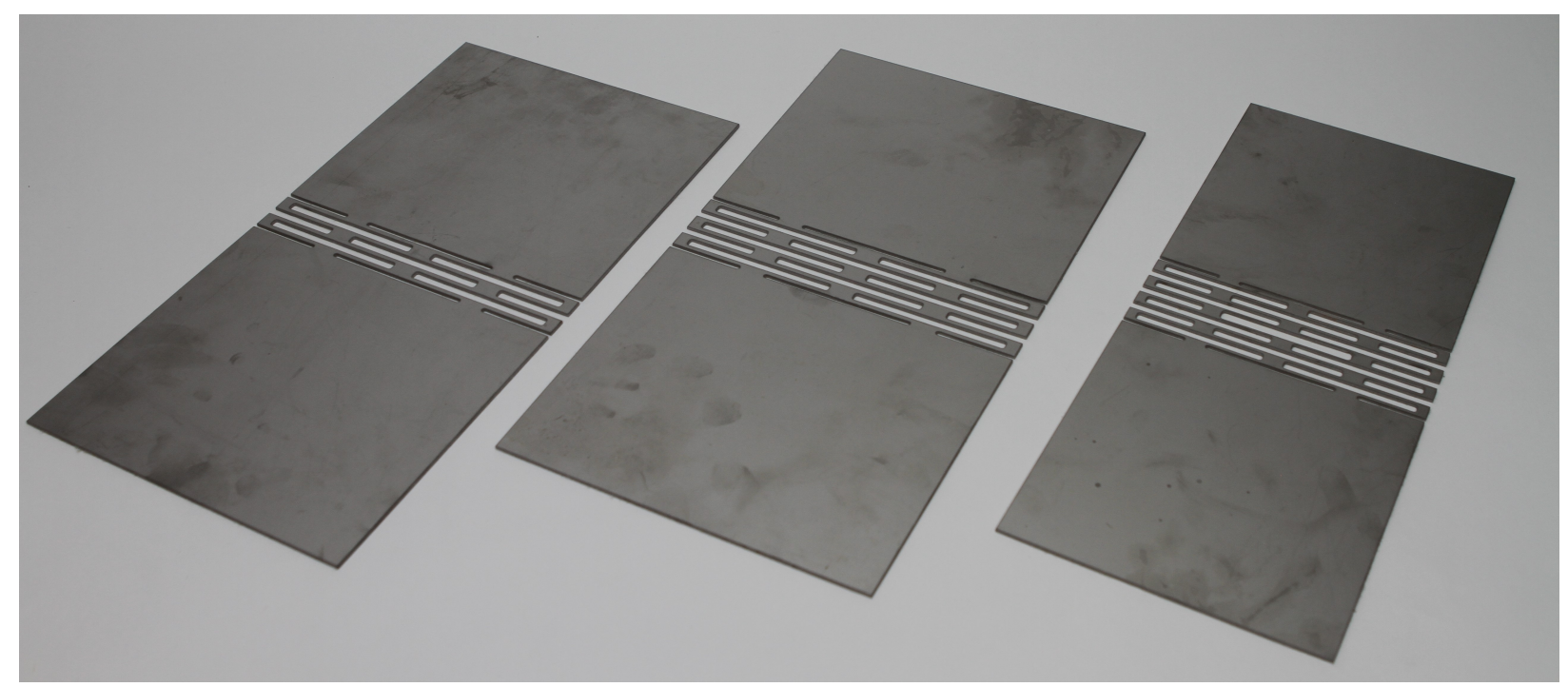

Figure 5: Three stainless steel prototypes of the Unidirectional CA: left $2 \mathrm{x} 2$ array, middle $3 \mathrm{x} 2$ array, right $4 \times 2$ array.

largest array, a 4x2 array, with different geometry parameters than the first two prototypes. These prototypes are shown in Figure 5.

A four-point bending test performed on a hydraulic tensile test machine (Instron Model 1321 with a $200 \mathrm{lb}$ load cell) was used to approximate a constant moment loading of the CAs across the y-axis. The rotational stiffness for each of the patterns, $K_{\text {uni,y }}$, was calculated using the elastic portion of the force displacement data from each of the tests as described next.

The moment imposed on the array by the four-point bending test, $M_{4 p o i n t}$, can be calculated as follows

$$
M_{4 p o i n t}=\frac{P L_{1}}{2}
$$

where $P$ is the measured applied force and $L_{1}$ is the length between the outside roller and closest middle roller, as shown in Figure 6.

The rotational deflection was calculated by assuming the stainless steel plate area without the CA to be rigid. This assumption is only appropriate when the bending stiffness of the $\mathrm{CA}$ is much less than that of the plate (a large shape factor), as is the case for each of the three geometries used in the prototypes. With this assumption the rotational deflection of the $\mathrm{CA}, \Theta$, can be computed from the measured linear deflection, $\delta$, using the following formula derived from the diagram in Figure 6.

$$
\Theta=2 \sin ^{-1} \frac{\delta}{L_{1}}
$$

where $L_{1}$ is the length between the outside roller and closest middle roller. 


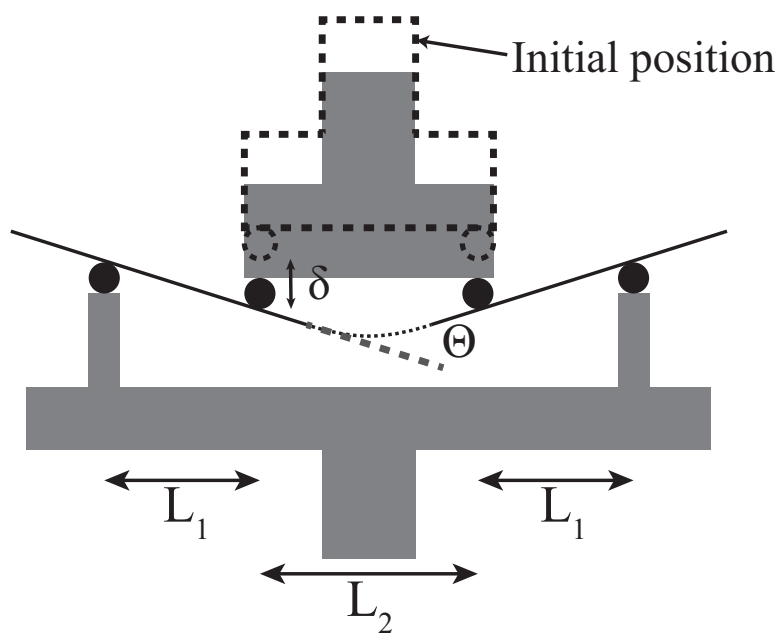

Figure 6: Four point bending test parameters.

Using Eqs. 38 and 39 and fitting a linear line to a plot of $M_{4 p o i n t}$ and $\Theta$ gives the stiffness of the array, $K_{u n i, y}$.

The reference stiffness of a panel without the CA, $S_{o}$, is calculated by Eqs. 18, 19, and 20. The flexural modulus, $E$, used in this equation was obtained for this particular stainless steel through a four-point bending test on a solid panel with the following equation derived from deflection equations from the shear moment diagrams:

$$
E=\left(\frac{P}{\delta}\right) \frac{L_{1}^{2}\left(2 L_{1}+3 L_{2}\right)}{12 I_{s}}
$$

where $P$ is the measured applied force, $L_{1}$ is the length between the outside and closest middle roller, $L_{2}$ is the distance between the two middle rollers, $I_{s}$ is the second moment of area for the sample in bending, and $\delta$ is the measured deflection. The measured modulus was $E=193 \mathrm{GPa}(28.0 \mathrm{Mpsi})$.

The experimental bending shape factors can now be computed using Eq. 18. The experimental shape factors for each of the three CAs are shown in Table 5 along with the analytically determined shape factors using the general shape factor equations from Table 1 .

The strength efficiency shape factor was also measured experimentally for the three arrays. The moment at failure, $M_{\max , y}$ was estimated from the recorded force-deflection plots by identifying the approximate force at yield and using Eq. 38. The maximum moment of the reference panel was calculated using Eq. 29 where $\sigma_{y}$, the yield stress, was measured from a four-point bend test on a solid panel using Eqs. 29 and 38. The measured yield stress was $254 \mathrm{MPa}(36.9 \mathrm{kpsi})$ for the stainless steel.

The experimental strength efficiency shape factors were computed using Eq. 2 for each of the three CAs. These experimental strength efficiency factors are shown in Table 5 with the analytically determined shape factors computed from Table 3 . The analytical and experimental results agree reasonably well. 
Table 5: Comparing experimentally determined and analytical shape factors for stainless steel Unidirectional CA prototypes.

\begin{tabular}{lcccc} 
& & & & \\
\cline { 4 - 5 } $2 \times 2$ array & Bending about y-axis & $\phi_{B}^{e}$ & 0.010 & 0.014 \\
& Strength Efficiency about y-axis & $\phi_{B}^{f}$ & 0.049 & 0.055 \\
\hline \multirow{2}{*}{$3 \times 2$ array } & Bending about y-axis & $\phi_{B}^{e}$ & 0.011 & 0.013 \\
& Strength Efficiency about y-axis & $\phi_{B}^{f}$ & 0.051 & 0.055 \\
\hline \multirow{2}{*}{$4 \times 2$ array } & Bending about y-axis & $\phi_{B}^{e}$ & 0.008 & 0.010 \\
& Strength Efficiency about y-axis & $\phi_{B}^{f}$ & 0.039 & 0.043 \\
\hline
\end{tabular}

\section{Results and Discussion}

\subsection{CA Shape Factor Analysis}

The elastic bending shape factors for the Unidirectional and Bidirectional arrays are summarized by the geometric parameters in Table 1. The strength efficiency shape factors are summarized for these two arrays in Table 3. Careful examination of the tables shows that the elastic bending shape factors for bending about the y-axis for the Unidirectional and Bidirectional CAs are nearly equivalent. The marked difference between the two arrays can be seen in bending about the $\mathrm{x}$-axis where the Bidirectional CA contains a larger denominator and thus smaller bending shape factor than the Unidirectional CA.

These shape factor expressions also show the independence of the shape factor from the scale of the shape. Multiplying all the geometric parameters by a constant scaling factor creates no variation in the expressions of the shape factors. Similarly, adding elements to the array in parallel and series as described by $m$ and $n$ has no effect on the shape factor as the shape of the fundamental unit remains the same. Only changing the ratio of geometric parameters to each other, and consequently changing the shape of the fundamental unit, causes the shape factor to change in value. This is seen in the results from the experimental prototypes as shown in Table 5 . The $2 \mathrm{x} 2$ and $3 \times 2$ arrays have nearly equivalent shape factors with small variation due to manufacturing tolerances that effected the shape slightly. The $4 \times 2$ array has different ratios of the geometric parameters and thus a different shape of the fundamental unit than the other prototypes. This differing shape is reflected in the change in the shape factor value.

It should be noted, as a small I-beam and larger scaled I-beam may share the same shape factor, the values of the stiffness and the load they can carry are not the same. Similarly, a CA with a larger scaled fundamental unit will have a larger stiffness and load carrying capacity than a CA with an equivalent shape factor on a smaller scale. The efficiency of the shapes in modifying the stiffness and strength are the same. This relationship is clearly seen in Equations 1 and 2 where the scale factors cancel out in the right side of the equations. However, if the equations are rearranged to find the stiffness and strength of a shape at a particular scale, the scale factor dictates the magnitude of the values. 
A range of shape factor values for CAs made of metals and polymers were generated by placing limits based on current manufacturing techniques and tolerances on the geometrical parameters used to define the arrays.

Laser cutting was found to be one of the most effective manufacturing methods in achieving the largest range of limits for these types of arrays. The parameters were then varied within these limits to establish a range of shape factors. In addition, a constraint was imposed to ensure the torsion bars would deflect in the intended torsion mode rather than a bending mode. This constraint can be described as $k_{b, u n i}>2 k_{t}$ for the Unidirectional array and $k_{b, b i}>2 k_{t}$ for the Bidirectional array. In these constraints $k_{t}$ is described by Eq. 6 and $k_{b, u n i}$ and $k_{b, b i}$ are the bending stiffness corresponding to the torsion bars for the Unidirectional array and Bidirectional array, respectively, and can be calculated for $w \geq t$ as

$$
\begin{gathered}
k_{b, \text { uni }}=\frac{E\left(4 b+2 L_{t}\right) t^{3}}{12 w} \\
k_{b, b i}=\frac{E\left(4 b+2 L_{t}+2 L_{b}\right) t^{3}}{12 w}
\end{gathered}
$$

The constraints can also be formulated for $t \geq w$ by swapping $t$ and $w$ in the above expressions and in Eq. 6.

An optimization routine was used to generate the extreme values for the shape factors with geometry still in the manufacturable ranges and satisfying the model assumption constraint. Table 6 lists the imposed limits, constraint equation, and the resulting shape factor values for a range of manufacturable arrays. It was seen that the length of the torsion bar, $L_{t}$, played the most dramatic role in determining the reduction of stiffness in bending about the y-axis. This was expected as $L_{t}$, most easily seen in the simplified shape factor equations, appears as a squared term in the denominator of the elastic bending shape factor expression about the y-axis whereas other parameters appear in a linear form. Similarly, when considering the strength efficiency shape factor for bending about the y-axis it is expected and was found that the parameter $w$, the width of the torsion bar, has the most dramatic impact in preserving bending strength.

Besides establishing the extreme values for the shape factors with a optimization routine, Latin hypercube sampling [50] of the five geometric parameters of the arrays was used to generate a visual depiction of the design space for both bending and strength efficiency factors. This space is shown in Figure 7 for bending about both the $\mathrm{y}$ and $\mathrm{x}$ axes for the Unidirectional array and in Figure 8 for the Bidirectional array.

These plots for the elastic bending shape factors and strength efficiency factors show that while the stiffness in a particular bending direction can be decreased by using the Unidirectional and Bidirectional arrays, the bending strength for that direction is also decreased. It also clear in the plots that the $\mathrm{x}$-direction bending shape factor is smaller for the Bidirectional array than the Unidirectional array. 

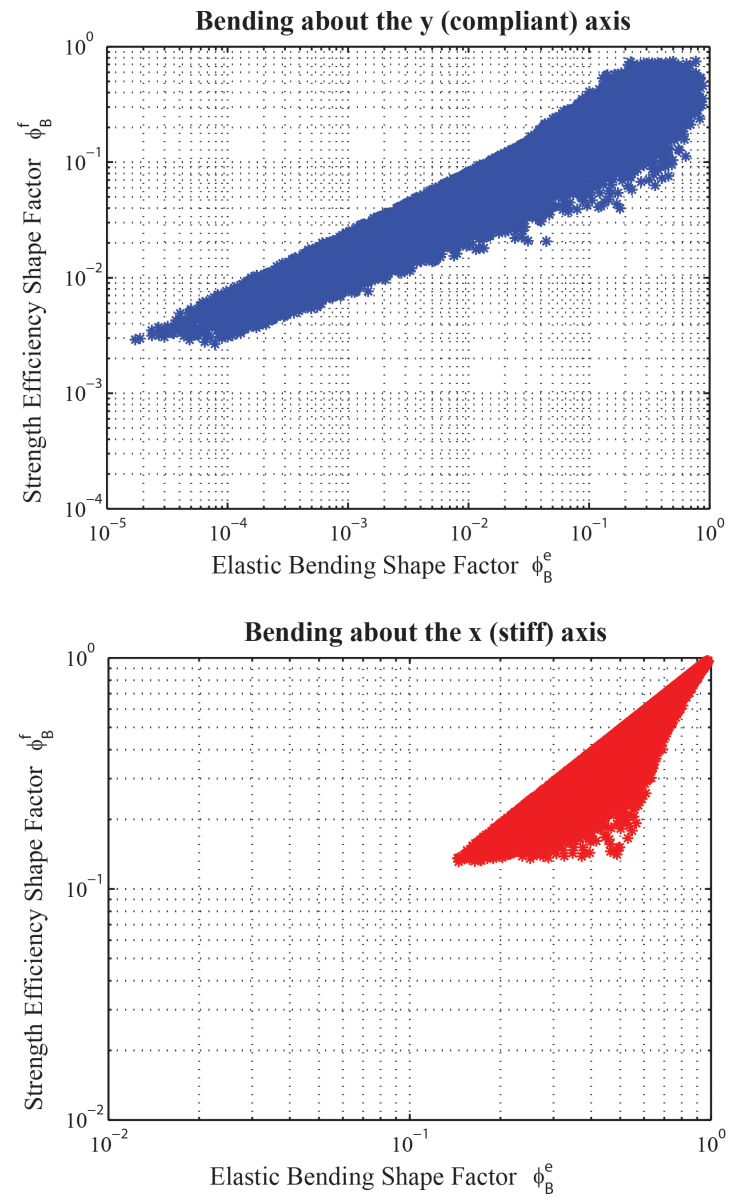

Figure 7: Shape Factor Design Space for Unidirectional Array. 

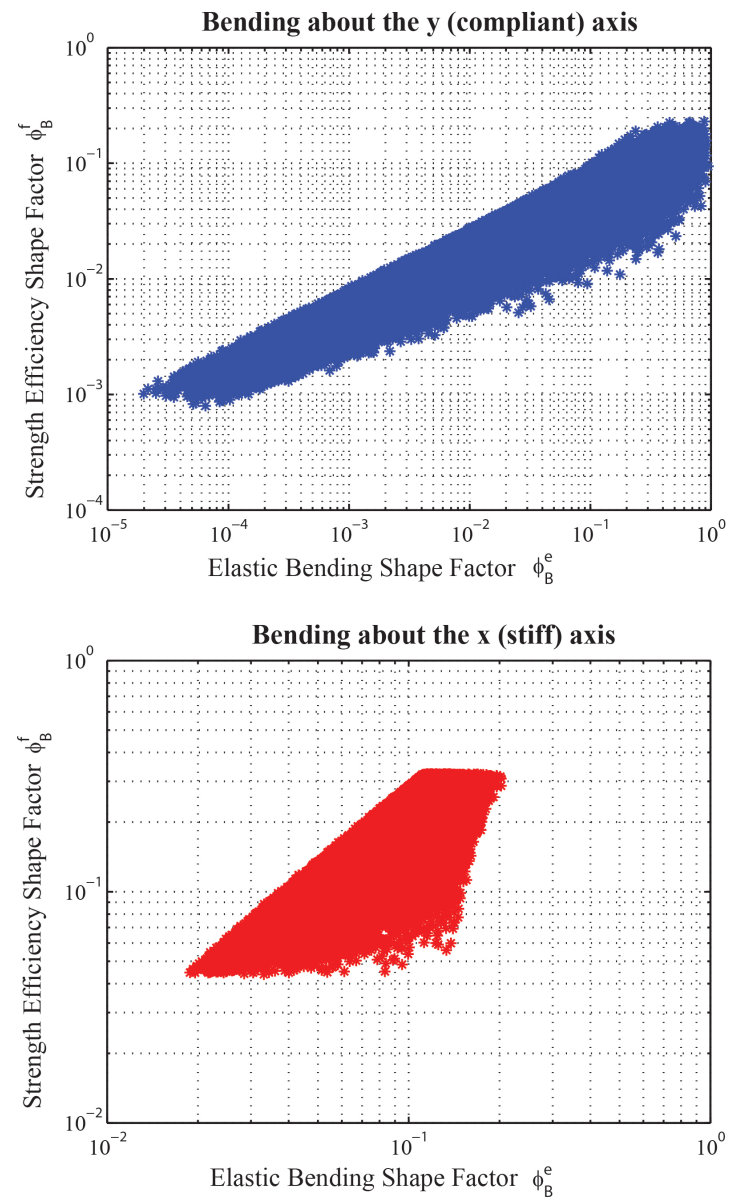

Figure 8: Shape Factor Design Space for Bidirectional Array. 
Table 6: Range of manufacturable shape factors, geometrical parameter limits, and constraints for metals and polymers.

\begin{tabular}{ccccc}
\hline \multicolumn{5}{c}{ Unidirectional Array Shape Factor Ranges } \\
\hline Geometric Parameters & Limits $(\mathrm{m})$ & Constraint & \multicolumn{2}{c}{ Shape Factor Ranges } \\
\hline$L_{b}$ & 0.0003 to 0.01 & $k_{b, u n i}>2 k_{t}$ & $\phi_{B}^{e}$ about y-axis & $1.26 \mathrm{e}-5$ to 0.964 \\
$b$ & 0.0015 to 0.01 & & $\phi_{B}^{e}$ about x-axis & 0.137 to 0.984 \\
$w$ & 0.0015 to 0.01 & & $\phi_{f}^{e}$ about y-axis & 0.0023 to 0.822 \\
$L_{t}$ & 0.0003 to 0.05 & $\phi_{f}^{e}$ about x-axis & 0.131 to 0.971 \\
$t$ & 0.0001 to 0.015 & Bidirectional Array Shape Factor Ranges \\
\hline \multicolumn{6}{c}{ Limits $(\mathrm{m})$} & Constraint & \multicolumn{3}{c}{ Shape Factor Ranges } \\
\hline$L_{b}$ & 0.0003 to 0.01 & $k_{b, b i}>2 k_{t}$ & $\phi_{B}^{e}$ about y-axis & $1.25 \mathrm{e}-5$ to 0.9837 \\
$w$ & 0.0015 to 0.01 & & $\phi_{B}^{e}$ about x-axis & 0.018 to 0.210 \\
$L_{t}$ & 0.0015 to 0.01 & & $\phi_{f}^{e}$ about y-axis & $6.83 \mathrm{e}-4$ to 0.2710 \\
$t$ & 0.0003 to 0.05 & $\phi_{f}^{e}$ about x-axis & 0.044 to 0.324 \\
\hline
\end{tabular}

\subsection{Material Selection with CAs}

The shape factors for the Unidirectional and Bidirectional CAs can be used in conjunction with Ashby charts of material properties to understand how the CA geometry affects the effective material properties of a component. Because CAs are generally employed to reduce bending stiffness, charts which include stiffness on at least one axis are most applicable for CA design. For example if a designer is concerned about mass and bending stiffness, the chart of Young's Modulus vs. Density can be used as shown in Figure 9. To see the effect of the elastic bending shape factor for bending about the y-axis of the Unidirectional CA, the chart can be modified by the corresponding shape factor and reference shape parameters. This is accomplished by scaling the axis with Young's modulus by the constant, $\frac{I_{0}}{L_{0}}$, where $I_{o}$ is the second moment of area of the reference shape about the bending axis and $L_{o}$ is the length of array perpendicular to the bending axis. By scaling this axis the chart now shows the stiffness of the reference panel, $\frac{E I_{o}}{L_{o}}$, for each material on the chart plotted against the density of the material. The shape factor for elastic bending about the y-axis can now be applied to the chart by taking any point of interest and multiplying the y coordinate, (the stiffness of the reference panel), by the shape factor. The resulting point is the stiffness of the CA made of the same material as the point of interest and with an equivalent cross-sectional area as the reference panel.

Using this method, the material envelopes of the Ashby chart can be expanded to show the effects of the shape factors on the effective material properties. The effects of the shape factor for elastic bending about the y-axis for the Unidirectional array according to the manufacturable range for metals and polymers are shown in Figure 10. It should be noted that while these expanded envelopes are accurate for metals and polymers, they are extrapolated to other types of materials, such as ceramics, where the ability to physically 


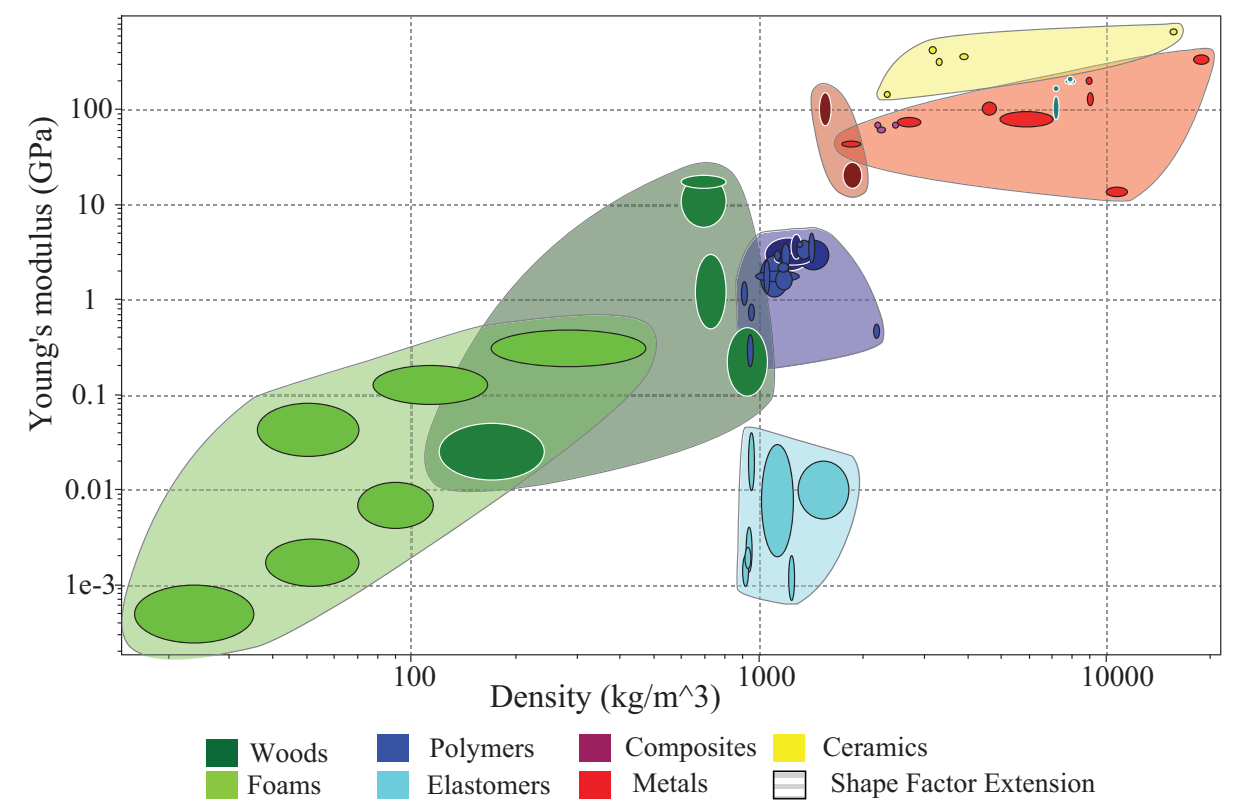

Figure 9: The Ashby chart of Young's modulus vs. density.

manufacture the CA geometry needs to be considered before using the chart.

While scaling the modulus axis of a material property chart by the constant $\frac{E I_{o}}{L_{o}}$ presents a straightforward representation of how the stiffness of a panel changes depending on the material and shape factor, it can also be useful to think of the shape factor as representing a "new" material with a modulus, $\phi_{B}^{e} E$. With this thought process a designer can look at a material property chart such as that in Figure 9 and plot a "new" material with modulus $\phi_{B}^{e} E$ and the original density, $\rho$.

The same type of approach can be employed to show the effect of the strength efficiency shape factor on the material property elastic yield strength for bending applications. The elastic yield strength can be multiplied by the constant $\frac{I_{o}}{c}$, where $I_{o}$ is the second moment of area of the reference shape about the bending axis and $c$ is the farthest distance from the neutral axis of the reference shape in bending. This scaling of the axis modifies the chart to show the maximum bending moment carried by the reference shape plotted against the density. The shape factor for strength efficiency can be applied in the same way as with the elastic bending. In addition, the CA again can be considered a "new" material with an effective yield strength of $\phi_{B}^{f} \sigma_{y}$ in bending. The modified Ashby chart of elastic yield strength plotted against density with the effects of the strength efficiency shape factors of the manufacturable range for polymers and metals in bending about the y-axis for the Unidirectional array is shown in Figure 11.

To maximize angular deflection of a panel, which is often the impetus for using a CA, a designer is interested in maximizing the ratio of yield strength over bending stiffness. For this case, the Ashby plot for yield strength vs. Young's modulus is used. Figure 12 shows how a single point on the chart can be moved about the material property space using various geometries of the Unidirectional $\mathrm{CA}$ within the manufacturable range for polymers 


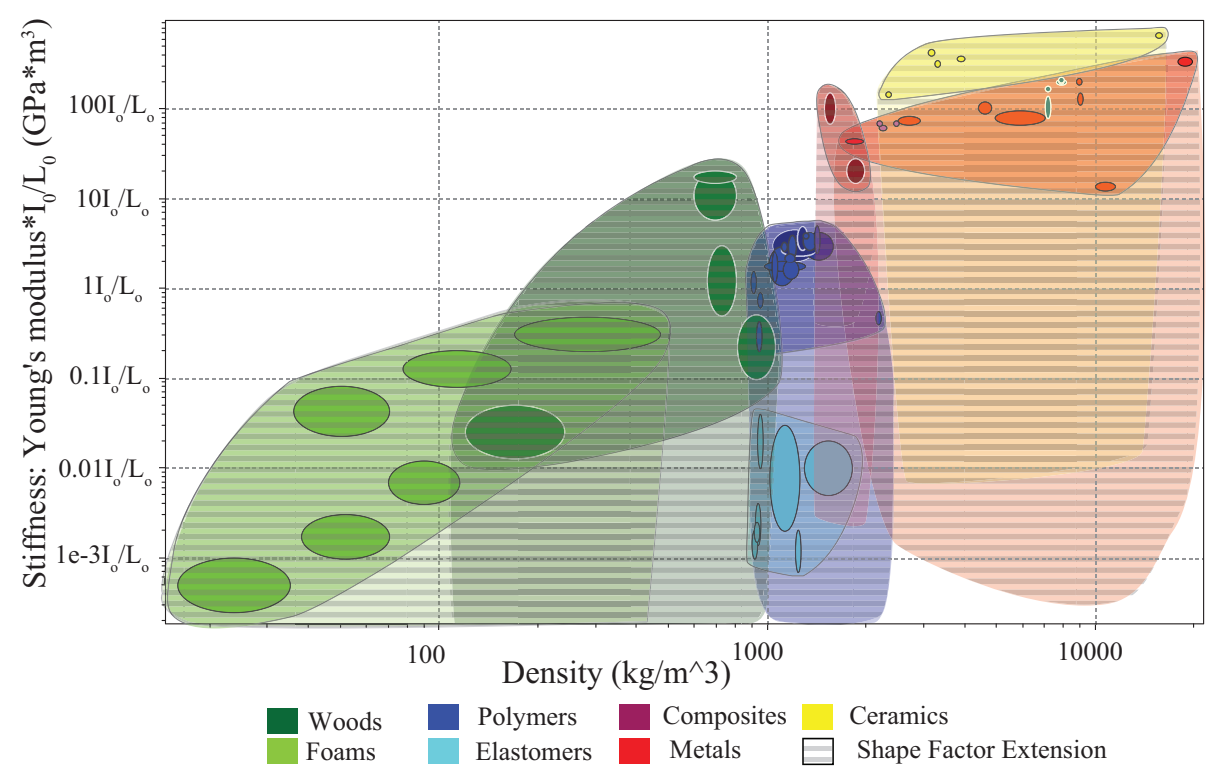

Figure 10: The Ashby chart of Young's modulus vs. density modified to show bending stiffness about the $\mathrm{y}$-axis for a panel and effects of the shape factor for the Unidirectional CA.

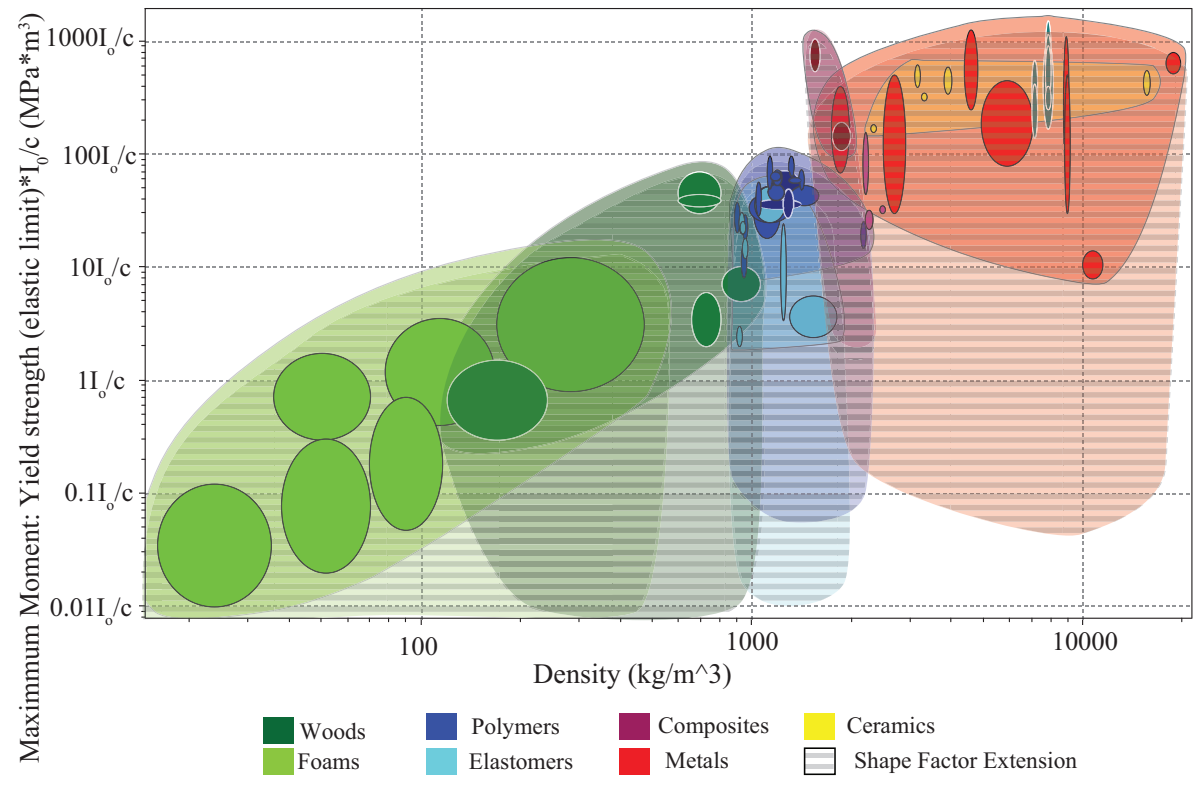

Figure 11: The Ashby chart of elastic yield strength vs. density modified to show the effect of the shape factor on the maximum moment withstood in bending about the y-axis for the Unidirectional CA. 


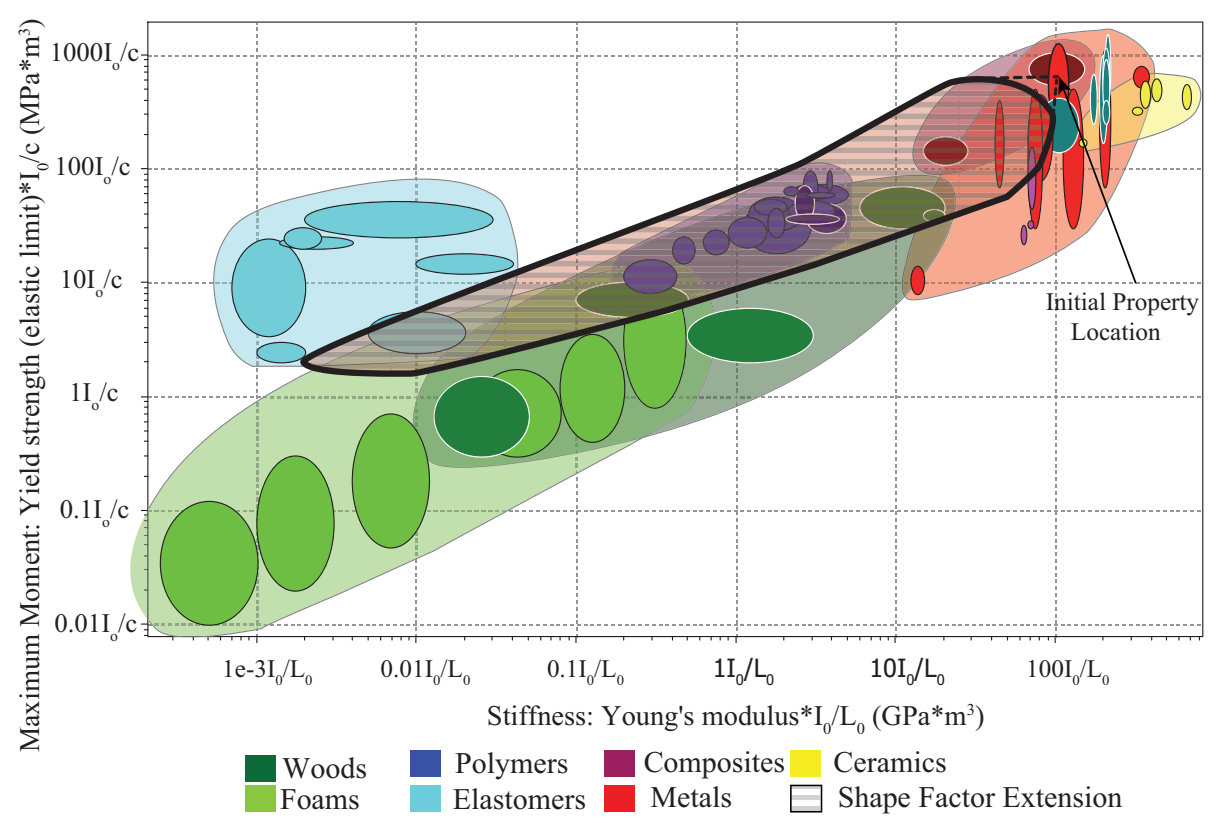

Figure 12: The Ashby chart of yield strength vs. Young's modulus with modified axes and examples of how single material property points can be moved about the chart as a consequence of using a Unidirectional $\mathrm{CA}$ in bending about the y-axis.

and metals in bending about the y-axis. The possible area to where the point can be moved in the material property space correlates with the possible shape factor space shown in Figure 7.

Other material property charts, such as the electrical conductivity vs. modulus chart in Figure 13, provide examples of how a reduction in stiffness by a CA can enable a designer to access previously empty sections of the material property charts. The electrical conductivity chart shows a trend where an increase in conductivity is accompanied by an increase in modulus. Here if a designer desires a flexible panel which is still electrically conductive, such as in a flexible circuit, a CA can be used to achieve this decrease in stiffness.

\section{Conclusions}

This work demonstrates how the notion of shape factors can be extended to flexible panels characterized by planar geometries. It describes how the specific geometry of two CAs, the Unidirectional and Bidirectional, can be captured in shape factors for elastic bending and strength efficiency. These shape factors help to show the tradeoff between a reduction in bending stiffness and a reduction in bending strength. Realistic ranges of these shape factors were determined and plotted on several Ashby material property charts. These charts show the previously inaccessible spaces which now can be accessed through the use of the compliant array. The designer can use the CA to decouple properties which are commonly linearly connected, such as electrical conductivity and stiffness. The expressions for the CA shape factors in conjunction with Ashby material charts provide a succinct way for designers 


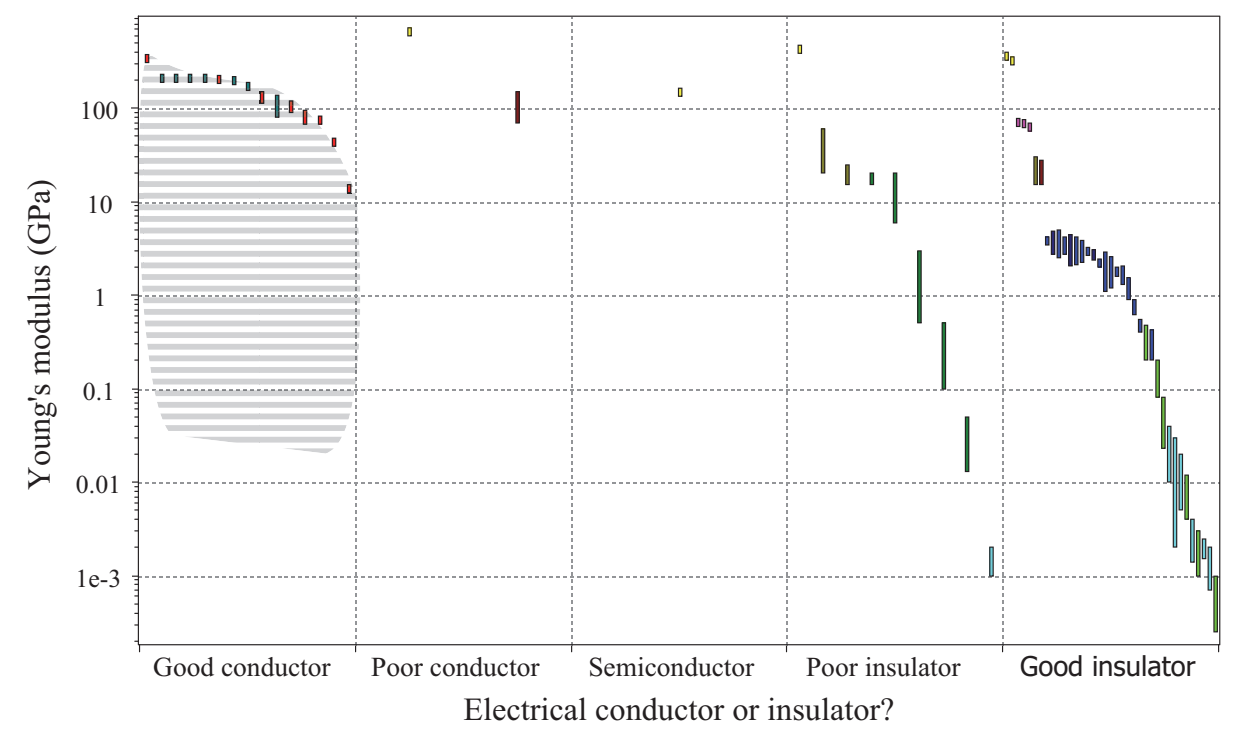

Figure 13: The Ashby chart of Young's modulus vs. qualitative electrical conductivity with the shaded region showing shape factor effects on good conductor panels shaped as Unidirectional CAs placed in bending about the y-axis.

to easily explore the benefits and drawbacks of using Unidirectional and Bidirectional CAs for particular bending applications.

\section{Acknowledgments}

This material is based on work supported by the National Science Foundation and the Air Force Office of Scientific Research under NSF Grant EFRI-ODISSEI-1240417. TG Nelson was supported by National Science Foundation Graduate Research Fellowship Program under Grant No. 1247046. DT Fullwood was supported by NSF grant CMMI 1538447. The authors would also like to acknowledge Kevin Cole and other Brigham Young University personnel for helping with the testing.

[1] J. O. Jacobsen, B. G. Winder, L. L. Howell, S. P. Magleby, Lamina emergent mechanisms and their basic elements, Journal of Mechanisms and Robotics 2 (1) (2010) 011003. doi:10.1115/1.4000523.

[2] A. Lamoureux, K. Lee, M. Shlian, S. R. Forrest, M. Shtein, Dynamic kirigami structures for integrated solar tracking, Nature Communications 6 (8092) (2015) -. doi:10.1038/ncomms9092.

[3] Z. Song, X. Wang, C. Lv, Y. An, M. Liang, T. Ma, D. He, Y.-J. Zheng, S.-Q. Huang, H. Yu, H. Jiang, Kirigami-based stretchable lithium-ion batteries, Scientific Reports 5 (10988) (2015) -. doi:10.1038/srep10988.

[4] Y. Zhang, Z. Yan, K. Nan, D. Xiao, Y. Liu, H. Luan, H. Fu, X. Wang, Q. Yang, J. Wang, W. Ren, H. Si, F. Liu, L. Yang, H. Li, J. Wang, X. Guo, H. Luo, L. Wang, Y. Huang, J. A. Rogers, A mechanically driven form of kirigami as a route to $3 \mathrm{D}$ mesostructures in micro/nanomembranes, Proceedings of the National Academy of Sciences 112 (38) (2015) 11757-11764. doi:10.1073/pnas.1515602112.

[5] S. Yang, I.-S. Choi, R. D. Kamien, Design of super-conformable, foldable materials via fractal cuts and lattice kirigami, MRS Bulletin 41 (02) (2016) 130-138. doi:10.1557/mrs.2016.5.

[6] J. Lee, K. Kim, J. Ju, D.-M. Kim, Compliant cellular materials with elliptical holes for extremely high positive and negative poisson's ratios, Journal of Engineering Materials and Technology 137 (1) (2014) 011001. doi:10.1115/1.4028317. 
[7] M. Eidini, Zigzag-base folded sheet cellular mechanical metamaterials, Extreme Mechanics Letters 6 (2016) 96 - 102. doi:10.1016/j.eml.2015.12.006.

[8] T. G. Nelson, R. J. Lang, N. A. Pehrson, S. P. Magleby, L. L. Howell, Facilitating deployable mechanisms and structures via developable lamina emergent arrays, Journal of Mechanisms and Robotics 8 (3) (2016) 031006-031006-10. doi:10.1115/1.4031901.

[9] X. Zheng, H. Lee, T. H. Weisgraber, M. Shusteff, J. DeOtte, E. B. Duoss, J. D. Kuntz, M. M. Biener, Q. Ge, J. A. Jackson, S. O. Kucheyev, N. X. Fang, C. M. Spadaccini, Ultralight, ultrastiff mechanical metamaterials, Science 344 (6190) (2014) 1373-1377. doi:10.1126/science.1252291.

[10] V. Dey, G. Zani, M. Colombo, M. D. Prisco, B. Mobasher, Flexural impact response of textile-reinforced aerated concrete sandwich panels, Materials \& Design 86 (2015) 187 - 197. doi:10.1016/j.matdes.2015.07.004.

[11] B. Chen, D. Yin, W. Ye, S. Lin, J. Fan, J. Gou, Fiber-continuous panel-pillar structure in insect cuticle and biomimetic research, Materials \& Design 86 (2015) 686 - 691. doi:10.1016/j.matdes.2015.07.102.

[12] I. L. Delimont, Compliant joints suitable for use as surrogate folds, Master's thesis (2014).

[13] J. Gattas, Z. You, Design and digital fabrication of folded sandwich structures, Automation in Construction 63 (2016) 79-87. doi:10.1016/j.autcon.2015.12.002.

[14] R. Mahshid, H. N. Hansen, K. L. Hojbjerre, Strength analysis and modeling of cellular lattice structures manufactured using selective laser melting for tooling applications, Materials \& Design (2016) In Pressdoi:10.1016/j.matdes.2016.05.020.

[15] X.-T. Wang, X.-W. Li, L. Ma, Interlocking assembled 3D auxetic cellular structures, Materials \& Design 99 (2016) 467 - 476. doi:10.1016/j.matdes.2016.03.088.

[16] T. George, V. S. Deshpande, H. N. Wadley, Mechanical response of carbon fiber composite sandwich panels with pyramidal truss cores, Composites Part A: Applied Science and Manufacturing 47 (2013) 31 - 40. doi:10.1016/j.compositesa.2012.11.011.

[17] I. Ullah, M. Brandt, S. Feih, Failure and energy absorption characteristics of advanced 3D truss core structures, Materials \& Design 92 (2016) 937 - 948. doi:10.1016/j.matdes.2015.12.058.

[18] Y. Klett, K. Drechsler, Designing technical tessellations, Origami5 (2011) 305-322.

[19] K. Saito, S. Pellegrino, T. Nojima, Manufacture of arbitrary cross-section composite honeycomb cores based on origami techniques, Journal of Mechanical Design 136 (5) (2014) 051011. doi:10.1115/1.4026824.

[20] T. Ohshima, T. Tachi, T. Hiroya, Y. Yamaguchi, Analysis and design of elastic materials formed using 2D repetitive slit pattern, in: International Association for Shell and Spatial Structures (IASS) Symposium 2015, 2015.

[21] M. Isobe, K. Okumura, Initial rigid response and softening transition of highly stretchable kirigami sheet materials, Scientific Reports 6 (24758) (2016) -. doi:10.1038/srep24758.

[22] M. Ashby, Overview no. 92: Materials and shape, Acta Metallurgica et Materialia 39 (6) (1991) 1025 - 1039. doi:10.1016/0956-7151(91)90189-8.

[23] D. Pasini, Shape transformers for material and shape selection of lightweight beams, Materials \& Design 28 (7) (2007) 2071 - 2079. doi:10.1016/j.matdes.2006.05.028.

[24] J. Singh, V. Mirjalili, D. Pasini, Integrated shape and material selection for single and multiperformance criteria, Materials \& Design 32 (5) (2011) 2909-2922. doi:10.1016/j.matdes.2010.11.076.

[25] M. F. Ashby, Materials Selection in Mechanical Design, 4th Edition, Elsevier, 2011.

[26] A. Amany, D. Pasini, Material and shape selection for stiff beams under non-uniform flexure, Materials \& Design 30 (4) (2009) 1110 - 1117. doi:10.1016/j.matdes.2008.06.029.

[27] L. L. Howell, Compliant mechanisms, John Wiley \& Sons, 2001.

[28] S. A. Zirbel, R. J. Lang, M. W. Thomson, D. A. Sigel, P. E. Walkemeyer, B. P. Trease, S. P. Magleby, L. L. Howell, Accommodating thickness in origami-based deployable arrays, Journal of Mechanical Design 135 (11) (2013) 111005. doi:10.1115/1.4025372.

[29] C. Quaglia, A. Dascanio, A. Thrall, Bascule shelters: A novel erection strategy for origami-inspired deployable structures, Engineering Structures 75 (2014) 276-287. doi:10.1016/j.engstruct.2014.06.003.

[30] Q. T. Aten, B. D. Jensen, S. H. Burnett, L. L. Howell, A self-reconfiguring metamorphic nanoin- 
jector for injection into mouse zygotes, Review of Scientific Instruments 85 (5) (2014) 055005. doi:10.1063/1.4872077.

[31] D. J. Struik, Lectures on classical differential geometry, Courier Dover Publications, 1961.

[32] D. Fuchs, S. Tabachnikov, More on paperfolding, American Mathematical Monthly (1999) 2735doi:10.2307/2589583

[33] J. P. Duncan, J. Duncan, Folded developables, Proceedings of the Royal Society of London. A. Mathematical and Physical Sciences 383 (1784) (1982) 191-205. doi:10.1098/rspa.1982.0126.

[34] C. J. Gantes, J. J. Connor, R. D. Logcher, Y. Rosenfeld, Structural analysis and design of deployable structures, Computers \& Structures 32 (3) (1989) 661-669. doi:10.1016/0045-7949(89)90354-4.

[35] J. Solomon, E. Vouga, M. Wardetzky, E. Grinspun, Flexible developable surfaces, in: Computer Graphics Forum, Vol. 31(5), Wiley Online Library, 2012, pp. 1567-1576. doi:10.1111/j.14678659.2012.03162.x.

[36] R. J. Lang, One ellipse to rule them all, The Fold (Origami USA).

[37] E. D. Demaine, M. L. Demaine, D. A. Huffman, D. Koschitz, T. Tachi, Designing curved-crease tessellations of lenses: qualitative properties of rulings, in: Proceedings of the 6th International Meeting on Origami in Science, Mathematics and Education (OSME 2014), Tokyo, Japan, 2014.

[38] R. D. Koschitz, Computational design with curved creases : David huffman's approach to paperfolding, Ph.D. thesis, Massachusetts Institute of Technology (2014).

[39] T. Tachi, Rigid-foldable thick origami, Origami5 (2010) 253-264.

[40] S. Felton, M. Tolley, E. Demaine, D. Rus, R. Wood, A method for building self-folding machines, Science 345 (6197) (2014) 644-646. doi:10.1126/science.1252610.

[41] P. Weaver, M. Ashby, Material limits for shape efficiency, Progress in Materials Science 41 (1) (1997) 61 - 128. doi:10.1016/S0079-6425(97)00034-0.

[42] R. Birmingham, B. Jobling, Material selection: Comparative procedures and the significance of form, in: International Conference on Lightweight Materials in Naval Architecture, The Royal Institution of Naval Architects, 1996.

[43] S. S. Tolman, I. L. Delimont, L. L. Howell, D. T. Fullwood, Material selection for elastic energy absorption in origami-inspired compliant corrugations, Smart Materials and Structures 23 (9) (2014) 094010.

[44] I. L. Delimont, S. P. Magleby, L. L. Howell, A family of dual-segment compliant joints suitable for use as surrogate folds, Journal of Mechanical Design 137 (9) (2015) 092302. doi:10.1115/1.4030875.

[45] J. O. Jacobsen, G. Chen, L. L. Howell, S. P. Magleby, Lamina emergent torsional (LET) joint, Mechanism and Machine Theory 44 (11) (2009) 2098-2109. doi:10.1016/j.mechmachtheory.2009.05.015.

[46] W. C. Young, R. G. Budynas, A. M. Sadegh, Roark's Formulas for Stress and Strain, 8th Edition, McGraw-Hill, 2012.

[47] S. Timoshenko, J. Goodier, Theory of elasticity, McGraw-Hill classic textbook reissue series, McGrawHill, 1969.

[48] W. D. Callister, D. G. Rethwisch, Materials science and engineering: an introduction, Vol. 7, Wiley New York, 2007.

[49] R. L. Norton, Machine Design An Integrated Approach Third Edition, 3rd Edition, Pearson, Prentice Hall, 2006, pg. 176.

[50] R. L. Iman, Latin Hypercube Sampling, John Wiley \& Sons, Ltd, 2008. doi:10.1002/9780470061596.risk0299. 